Article

\title{
Morphology and Multi-Gene Phylogeny Reveal Pestalotiopsis pinicola sp. nov. and a New Host Record of Cladosporium anthropophilum from Edible Pine (Pinus armandii) Seeds in Yunnan Province, China
}

\author{
Saowaluck Tibpromma ${ }^{1,2}$, Peter E. Mortimer ${ }^{2, *}$, Samantha C. Karunarathna ${ }^{2}$, \\ Fangdong Zhan ${ }^{1}{ }^{1}$, Jianchu $\mathrm{Xu}^{2}$, Itthayakorn Promputtha ${ }^{3,4}$ and Kai Yan ${ }^{1,2, *}$ \\ 1 College of Resources and Environment, Yunnan Agricultural University, Kunming 650201, Yunnan, China; \\ saowaluckfai@gmail.com (S.T.); zfd97@ynau.edu.cn (F.Z.) \\ 2 Key Laboratory for Plant Diversity and Biogeography of East Asia, Kunming Institute of Botany, Chinese \\ Academy of Science, Kunming 650201, Yunnan, China; samanthakarunarathna@gmail.com (S.C.K.); \\ jxu@mail.kib.ac.cn (J.X.) \\ 3 Department of Biology, Faculty of Science, Chiang Mai University, Chiang Mai 50200, Thailand; \\ ppam118@hotmail.com \\ 4 Center of Excellence in Bioresources for Agriculture, Industry and Medicine, Department of Biology, \\ Faculty of Science, Chiang Mai University, Muang District, Chiang Mai 50200, Thailand \\ * Correspondence: peter@mail.kib.ac.cn (P.T.E.); yankai@mail.kib.ac.cn (K.Y.)
}

Received: 17 October 2019; Accepted: 30 November 2019; Published: 4 December 2019

\begin{abstract}
This study contributes new knowledge on the diversity of conidial fungi in edible pine (Pinus armandii) seeds found in Yunnan Province, China and emphasizes the importance of edible seed products to ensure food safety standards. We isolated two fungal species, one on the pine seed coat and the other on the endosperm of the pine seed. The two fungal species were identified as Pestalotiopsis pinicola sp. nov. and a new host record Cladosporium anthropophilum. Characteristic morphological features of Pestalotiopsis pinicola were used alongside results from multi-gene phylogenetic analysis to distinguish it from currently known species within the genus. Cladosporium anthropophilum was identified as a new host record based on morphological features and phylogenetic analysis. In addition, detailed descriptions, scanned electron microscopy morphology, illustrations, and phylogenetic trees are provided to show the placement of these species.
\end{abstract}

Keywords: ascomycota; endophytic fungi; new taxon; saprobic fungi; taxonomy; weak pathogen

\section{Introduction}

Chinese white pine (Pinus armandii), one of the endemic conifer species of East Asia, is known throughout China, and particularly Yunnan Province, for its substantial ecological and economic value $[1,2]$. Pinus armandii seeds are suitable for use as a culinary ingredient after roasting, because the fatty acid profile of the seeds has a higher level of taxoleic acid and lower levels of octadecenoic acids compared to other species in Pinus [3,4].

Seeds are colonized by various types of fungi including fungal pathogens [5]. Several fungal species exist in seeds in the forms of spores and mycelium and can subsist for long periods of time on the seed coat and in the inner areas [6]. In general, fungi that are present within seeds are more harmful than those that merely contaminate the outer seed coat [6]. Common fungi genera that have been reported as associated with various seeds are Aspergillus, Mucor, Penicillium, Pestalotiopsis, Rhizopus, 
and Trichoderma [7]. Some studies have confirmed that fungi that are usually thought to be saprobes act as pathogens under certain circumstances, while endophytes can also switch to a saprobic lifestyle $[8,9]$. Fungal invasions happen after injury to the seed or seed coat as well as when moisture levels and temperatures are favorable for fungal growth [10]. Many seed fungi are also important sources of bioactive compounds [11,12]. In this study, we were able to isolate and identify two micro-fungi belonging to the genera Cladosporium and Pestalotiopsis from the seeds of Pinus armandii.

The genus Cladosporium (Cladosporiaceae, Capnodiales) was introduced by Link [13] with C. herbarum (Pers.) Link as the type species. The members of this genus can be endophytes, pathogens, and saprobes with worldwide distribution across a wide range of disparate substrates [14-18]. Cladosporium species are also known as the most abundant fungi in indoor and outdoor environments and are also important as spoilage organisms and discoloration which have been screened from cereal grains, fruits, peanuts, and chilled meat [19-22]. While Cladosporium species have not been reported as mycotoxin producers, they may nonetheless represent a health threat. Furthermore, some species have been reported causing fungal allergies, especially in patients with severe asthma [23-31]. Recently, several Cladosporium species have been reported in China, Thailand, and the United Kingdom on the decaying seed pods of Delonix regia, Entada phaseoloides, Laburnum anagyroides, and Magnolia grandiflora [32]. Only two species, Cladosporium nigrellum and C. psoraleae, have been reported from Pinus armandii in China [33,34].

The genus Pestalotiopsis (Sporocadaceae, Amphisphaeriales) was introduced by Steyaert [35] with P. guepinii (Desm.) Steyaert as the type species. The members of this genus can be found worldwide as endophytes, saprobes or opportunistic pathogens [18,36-44]. Some of them are confirmed to cause human and animal diseases $[42,45,46]$. For example, Pestalotiopsis spp. have been isolated from a bronchial biopsy, corneal abrasions, eyes, feet, fingernails, scalp, and sinuses from the human body [45]. In addition, this genus is known as one of the common fungi genera reported on various seeds [7]. Pestalotiopsis algeriensis, P. carveri, P. caudata, P. cocculi, P. disseminate, P. heterocornis, P. lespedezae, P. neglecta, P. olivacea, and P. vismiae have been reported from Pinus armandii in China $[33,34,38,47]$.

In the present study, we used multi-gene sequence analysis, morphological examinations, and culture characteristics for the identification and delimitation of fungi isolates belonging to the genera Cladosporium and Pestalotiopsis from seeds of Pinus armandii collected in Yunnan Province, China.

\section{Materials and Methods}

\subsection{Sample Collection and Specimen Examination}

Fresh fungal structures (mycelia and spore masses) were directly isolated in potato dextrose agar (PDA) from seed coats and endosperms of Pinus armandii seeds using aseptic techniques, and the PDA plates were incubated at room temperature. Pine seeds were obtained outside Kunming, Yunnan Province, China (Figure 1). The seeds were then carefully analyzed. Morphological structures of the fungi were examined under a stereo microscope. Scanning electron microscopy (SEM) micrographs were obtained under a ZEISS GeminiSEM and ZEISS Sigma 300 apparatus, following the methods described by Figueras and Guarro [48]. To observe the fungal structures, sporulated cultures were mounted on water. Microscopic fungal structures were observed under a compound microscope and photographs were captured with a digital camera fitted on to the microscope. All microscopic structures of fungi were measured by the Tarosoft Image Framework program v.0.9.0.7., and Adobe Photoshop CS3 Extended version 10.0 (Adobe Systems, USA) was used to process and edit the images used in the figures.

Isolation

The PDA medium was used for culturing the isolated fungi. Spore masses from the seed coat and mycelia from the endosperm were aseptically transferred to PDA plates (two isolates of each species). The pure culture plates were incubated at room temperature $\left(20-25^{\circ} \mathrm{C}\right)$ for $14-21$ days, and 
the fungal colonies were carefully observed and described. The herbarium specimens of the fungi were dehydrated using silica gel and deposited in the Mae Fah Luang University Herbarium. The pure cultures were deposited in the Kunming Institute of Botany Culture Collection (KMUCC). Index Fungorum (IF) and Facesoffungi (FoF) numbers were obtained as described by Index Fungorum [49] and Jayasiri et al. [50].
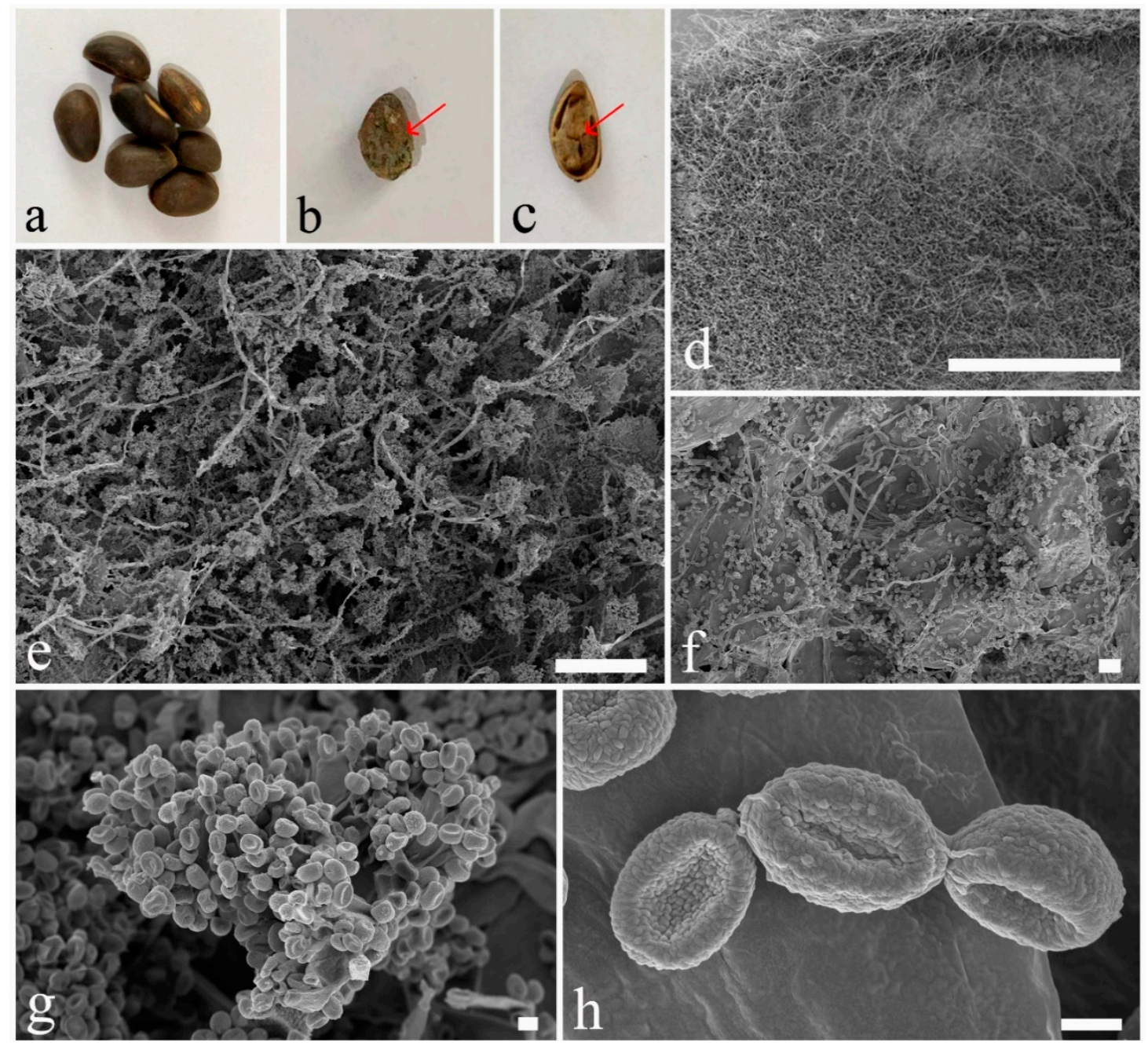

Figure 1. (a) Seeds of Pinus armandii from Yunnan Province, China. (b) Fungal mass on a seed. (c) Endosperm covered with mycelia. (d) Mycelia on endosperm under a SEM micrograph. (e-h) Rows of rounded cells present on a seed coat skin under SEM micrographs that form aerial hyphae, conidia, and conidiophores. Scale bars: $\mathrm{d}=1 \mathrm{~mm}, \mathrm{e}=100 \mu \mathrm{m}, \mathrm{f}=10 \mu \mathrm{m}, \mathrm{g}=2 \mu \mathrm{m}, \mathrm{h}=1 \mu \mathrm{m}$.

\subsection{DNA Extraction, PCR Amplification, and DNA Sequencing}

The mycelia of the cultures grown on PDA at room temperature for 4 weeks were used for DNA extraction. The fungal mycelia were scraped off with a sterile scalpel and transferred to $1.5 \mathrm{~mL}$ micro-centrifuge tubes under aseptic conditions and kept at $-20{ }^{\circ} \mathrm{C}$ to avoid contaminations until use. The Biospin Fungal Genomic DNA Extraction Kit (BioFlux, China) was used to perform DNA extraction from the fungal cultures, following the manufacturer's protocols. To amplify partial gene regions of the 5.8S rRNA gene in the internal transcribed spacer (ITS), translation elongation factor 1-alpha gene (TEF1), actin gene (ACT), and beta-tubulin gene (TUB2), polymerase chain reaction (PCR) was used. The PCR conditions and primers were set under standard conditions as shown in Table 1. The total volume of PCR mixtures for amplifications was set as described in Tibpromma et al. [18]. Purification and sequencing of PCR products were done by Sangon Biotech Co., Shanghai, China. 
Table 1. Gene regions and primers used in this study.

\begin{tabular}{ccc}
\hline Genes & Primers (Forward/Reverse) & References \\
\hline Cladosporium & \\
\hline ACT & $512 \mathrm{~F} / 783 \mathrm{R}$ & {$[51]$} \\
\hline TEF1 & ITS5/ITS4 & {$[52]$} \\
\hline ITS & Pestalotiopsis & \\
\hline TEF1 & ITS5/ITS4 & {$[52]$} \\
\hline TUB2 & 526F/1567R & {$[53]$} \\
\hline & T1/T2 & {$[54]$} \\
\hline
\end{tabular}

\subsection{Phylogenetic Analyses}

The ITS and TEF1 sequence data produced in this study were used in BLAST searches in the GenBank database (www.http://blast.ncbi.nlm.nih.gov/) to determine their most probable closely related taxa. The sequence data generated in this study were analyzed with closely related taxa retrieved from GenBank based on BLAST searches and recent publications $[9,16,18,55,56]$. Single gene sequence datasets were aligned using the MAFFT v.7.215 website [57] and manually edited in BioEdit v.7.0 when necessary [58]. Single sequence alignment datasets were combined using BioEdit v.7.2.5 [58]. The alignment of combined datasets in FASTA format was converted to PHYLIP and NEXUS formats using the Alignment Transformation Environment (ALTER) website [59]. Phylogenetic trees were run in randomized accelerated maximum likelihood (RAxML) and Bayesian posterior probabilities (BYPPs). The maximum likelihood (ML) analysis was performed via the CIPRES Science Gateway [60] using the RAxML-HPC BlackBox (8.2.4) section [61,62] with the general time reversible model (GTR) using a discrete gamma distribution as the evolutionary model. To carry out Bayesian analysis, the model of evolution was estimated using MrModeltest 2.2 [63] with HKY+I+G (for the Pestalotiopsis dataset) and GTR $+\mathrm{I}+\mathrm{G}$ (for the Cladosporium dataset) as nucleotide substitution models selected for combined datasets. Posterior probabilities (PPs) [64] were determined by Markov chain Monte Carlo sampling (MCMC) in MrBayes v.3.0b4 [65]. The parameters were set as six simultaneous Markov chains ran for 5,000,000 generations and sampling every 100th generation for a total of 50,000 trees [66]. The first trees representing the burn-in phase of the analysis $(20 \%)$ were discarded and the remaining (post-burn) trees were used for calculating PPs in the majority rule consensus tree (the critical value for the topological convergence diagnostic values reached 0.01) [67,68].

The phylograms were figured in FigTree v.1.4 [69] and reorganized using Microsoft Office PowerPoint 2007 and Adobe illustrator CS3 (Adobe Systems Inc., USA). The sequences generated in this study were submitted to GenBank (Tables 2 and 3).

Table 2. GenBank accession numbers and culture collection numbers of the nucleotide sequences of Cladosporium taxa used in this study. The new sequences generated in this study are in bold type.

\begin{tabular}{lllll}
\hline \multirow{2}{*}{ Species } & \multirow{2}{*}{ Culture Collection Number } & \multicolumn{2}{c}{ GenBank Accession Numbers } \\
\cline { 3 - 5 } & & \multicolumn{1}{c}{ ITS } & \multicolumn{1}{c}{ TEF1 } & ACT \\
\hline Cercospora beticola & CBS 116456 & NR_121315 & AY840494 & AY840458 \\
Cladosporium acalyphae & CBS 125982 & HM147994 & HM148235 & HM148481 \\
C. alboflavescens & CBS 140690 & LN834420 & LN834516 & LN834604 \\
C. angustisporum & CBS 125983 & HM147995 & HM148236 & HM148482 \\
C. angustisporum & UTHSC-DI-13-240 & LN834356 & LN834452 & LN834540 \\
C. anthropophilum & CBS 117483 & HM148007 & HM148248 & HM148494 \\
C. anthropophilum & CBS 140685 & LN834437 & LN834533 & LN834621 \\
C. anthropophilum & KUMCC 19-0182 & MN412638 & MN417513 & MN417511 \\
\hline
\end{tabular}


Table 2. Cont.

\begin{tabular}{|c|c|c|c|c|}
\hline \multirow{2}{*}{ Species } & \multirow{2}{*}{ Culture Collection Number } & \multicolumn{3}{|c|}{ GenBank Accession Numbers } \\
\hline & & ITS & TEF1 & $A C T$ \\
\hline C. anthropophilum & KUMCC 19-0202 & MN412639 & MN417514 & MN417512 \\
\hline C. anthropophilum & UTHSC-DI-13-168 & LN834407 & LN834503 & LN834591 \\
\hline C. anthropophilum & UTHSC-DI-13-169 & LN834408 & LN834504 & LN834592 \\
\hline C. anthropophilum & UTHSC-DI-13-178 & LN834410 & LN834506 & LN834594 \\
\hline C. anthropophilum & UTHSC-DI-13-179 & LN834411 & LN834507 & LN834595 \\
\hline C. anthropophilum & UTHSC-DI-13-207 & LN834413 & LN834509 & LN834597 \\
\hline C. anthropophilum & UTHSC-DI-13-226 & LN834421 & LN834517 & LN834605 \\
\hline C. anthropophilum & UTHSC-DI-13-228 & LN834423 & LN834519 & LN834607 \\
\hline C. anthropophilum & UTHSC-DI-13-244 & LN834428 & LN834524 & LN834612 \\
\hline C. anthropophilum & UTHSC-DI-13-246 & LN834430 & LN834526 & LN834614 \\
\hline C. anthropophilum & UTHSC-DI-13-269 & LN834437 & LN834533 & LN834621 \\
\hline C. anthropophilum & UTHSC-DI-13-271 & LN834439 & LN834535 & LN834623 \\
\hline C. asperulatum & CBS 126339 & HM147997 & HM148238 & HM148484 \\
\hline C. asperulatum & CBS 126340 & HM147998 & HM148239 & HM148485 \\
\hline C. australiense & CBS 125984 & HM147999 & HM148240 & HM148486 \\
\hline C. austroafricanum & СРC 16763 & KT600381 & KT600478 & KT600577 \\
\hline C. chalastosporoides & CBS 125985 & HM148001 & HM148242 & HM148488 \\
\hline C. chubutense & CBS 124457 & FJ936158 & FJ936161 & FJ936165 \\
\hline C. cladosporioides & CBS 112388 & HM148003 & HM148244 & HM148490 \\
\hline C. cladosporioides & CBS 113738 & HM148004 & HM148245 & HM148491 \\
\hline C. cladosporioides & CPC 14292 & HM148046 & HM148287 & HM148533 \\
\hline C. cladosporioides & UTHSC-DI-13-215 & LN834360 & LN834456 & LN834544 \\
\hline C. colocasiae & CBS 119542 & HM148066 & HM148309 & HM148554 \\
\hline C. colocasiae & CBS 386.64 & HM148067 & HM148310 & HM148555 \\
\hline C. colombiae & CBS 274.80B & FJ936159 & FJ936163 & FJ936166 \\
\hline C. crousii & UTHSC-DI-13-247 & LN834431 & LN834527 & LN834615 \\
\hline C. сиситеrinum & CBS 171.52 & HM148072 & HM148316 & HM148561 \\
\hline C. cucumerinum & CBS 173.54 & HM148074 & HM148318 & HM148563 \\
\hline C. delicatulum & CBS 126342 & HM148079 & HM148323 & HM148568 \\
\hline C. delicatulum & CBS 126344 & HM148081 & HM148325 & HM148570 \\
\hline C. exile & CBS 125987 & HM148091 & HM148335 & HM148580 \\
\hline C. flabelliforme & CBS 126345 & HM148092 & HM148336 & HM148581 \\
\hline C. flabelliforme & UTHSC-DI-13-267 & LN834361 & LN834457 & LN834545 \\
\hline C. flavovirens & UTHSC-DI-13-273 & LN834440 & LN834536 & LN834624 \\
\hline C. funiculosum & CBS 122128 & HM148093 & HM148337 & HM148582 \\
\hline C. funiculosum & CBS 122129 & HM148094 & HM148338 & HM148583 \\
\hline C. gamsianum & CBS 125989 & HM148095 & HM148339 & HM148584 \\
\hline C. globisporum & CBS 812.96 & HM148096 & HM148340 & HM148585 \\
\hline C. grevilleae & CBS 114271 & JF770450 & JF770472 & JF770473 \\
\hline C. hillianum & CBS 125988 & HM148097 & HM148341 & HM148586 \\
\hline C. inversicolor & CBS 143.65 & HM148100 & HM148344 & HM148589 \\
\hline C. ipereniae & CBS 140483 & KT600394 & KT600491 & KT600589 \\
\hline C. ipereniae & СРC 16855 & KT600395 & KT600492 & KT600590 \\
\hline C. iranicum & CBS 126346 & HM148110 & HM148354 & HM148599 \\
\hline C. limoniforme & CBS 113737 & KT600396 & KT600493 & KT600591 \\
\hline C. longicatenatum & СРС 17189 & KT600403 & KT600500 & KT600598 \\
\hline C. lycoperdinum & CBS 126347 & HM148112 & HM148356 & HM148601 \\
\hline C. lycoperdinum & CBS 574.78C & HM148115 & HM148359 & HM148604 \\
\hline C. montecillanum & СРС 15605 & KT600407 & KT600505 & KT600603 \\
\hline C. montecillanum & СРC 17953 & KT600406 & KT600504 & KT600602 \\
\hline C. myrtacearum & CBS 126350 & HM148117 & HM148361 & HM148606 \\
\hline C. oxysporum & CBS 125991 & HM148118 & HM148362 & HM148607 \\
\hline C. oxysporum & CBS 126351 & HM148119 & HM148363 & HM148608 \\
\hline C. paracladosporioides & CBS 171.54 & HM148120 & HM148364 & HM148609 \\
\hline C. parapenidielloides & СРC 17193 & KT600410 & KT600508 & KT600606 \\
\hline
\end{tabular}


Table 2. Cont.

\begin{tabular}{|c|c|c|c|c|}
\hline \multirow{2}{*}{ Species } & \multirow{2}{*}{ Culture Collection Number } & \multicolumn{3}{|c|}{ GenBank Accession Numbers } \\
\hline & & ITS & TEF1 & $A C T$ \\
\hline C. phaenocomae & CBS 128769 & JF499837 & JF499875 & JF499881 \\
\hline C. phyllactiniicola & CBS 126353 & HM148151 & HM148395 & HM148640 \\
\hline C. phyllactiniicola & CBS 126355 & HM148153 & HM148397 & HM148642 \\
\hline C. phyllophilum & CBS 125992 & HM148154 & HM148398 & HM148643 \\
\hline C. phyllophilum & CBS 125990 & HM148111 & HM148355 & HM148600 \\
\hline C. pini-ponderosae & CBS 124456 & FJ936160 & FJ936164 & FJ936167 \\
\hline C. pseudochalastosporoides & СРC 17823 & KT600415 & KT600513 & KT600611 \\
\hline C. pseudocladosporioides & CBS 125993 & HM148158 & HM148402 & HM148647 \\
\hline C. pseudocladosporioides & CBS 667.80 & HM148165 & HM148409 & HM148654 \\
\hline C. pseudocladosporioides & CPC 13683 & HM148173 & HM148417 & HM148662 \\
\hline C. pseudocladosporioides & СРC 14020 & HM148185 & HM148429 & HM148674 \\
\hline C. pseudocladosporioides & CРC 14295 & HM148188 & HM148432 & HM148677 \\
\hline C. pseudocladosporioides & UTHSC-DI-13-165 & LN834406 & LN834502 & LN834590 \\
\hline C. pseudocladosporioides & UTHSC-DI-13-190 & LN834412 & LN834508 & LN834596 \\
\hline C. pseudocladosporioides & UTHSC-DI-13-210 & LN834414 & LN834510 & LN834598 \\
\hline C. pseudocladosporioides & UTHSC-DI-13-218 & LN834418 & LN834514 & LN834602 \\
\hline C. pseudocladosporioides & UTHSC-DI-13-227 & LN834422 & LN834518 & LN834606 \\
\hline C. pseudocladosporioides & UTHSC-DI-13-234 & LN834424 & LN834520 & LN834608 \\
\hline C. pseudocladosporioides & UTHSC-DI-13-238 & LN834426 & LN834522 & LN834610 \\
\hline C. pseudocladosporioides & UTHSC-DI-13-241 & LN834427 & LN834523 & LN834611 \\
\hline C. pseudocladosporioides & UTHSC-DI-13-245 & LN834429 & LN834525 & LN834613 \\
\hline C. pseudocladosporioides & UTHSC-DI-13-251 & LN834432 & LN834528 & LN834616 \\
\hline C. pseudocladosporioides & UTHSC-DI-13-261 & LN834384 & LN834480 & LN834568 \\
\hline C. pseudocladosporioides & UTHSC-DI-13-265 & LN834435 & LN834531 & LN834619 \\
\hline C. pseudocladosporioides & UTHSC-DI-13-268 & LN834436 & LN834532 & LN834620 \\
\hline C. pseudocladosporioides & UTHSC-DI-13-270 & LN834438 & LN834534 & LN834622 \\
\hline C. rectoides & CBS 125994 & HM148193 & HM148438 & HM148683 \\
\hline C. ruguloflabelliforme & СРС 19707 & KT600458 & KT600557 & KT600655 \\
\hline C. scabrellum & CBS 126358 & HM148195 & HM148440 & HM148685 \\
\hline C. silenes & CBS 109082 & EF679354 & EF679429 & EF679506 \\
\hline C. subinflatum & CBS 121630 & EF679389 & EF679467 & EF679543 \\
\hline C. subinflatum & CBS 121630 & EF679389 & EF679467 & EF679543 \\
\hline C. subuliforme & CBS 126500 & HM148196 & HM148441 & HM148686 \\
\hline C. tenuissimum & СРC 13222 & HM148210 & HM148455 & HM148700 \\
\hline C. tenuissimum & CPC 14250 & HM148211 & HM148456 & HM148701 \\
\hline C. tenuissimum & UTHSC-DI-13-258 & LN834404 & LN834500 & LN834588 \\
\hline C. varians & CBS 126362 & HM148224 & HM148470 & HM148715 \\
\hline C. verrucocladosporioides & CBS 126363 & HM148226 & HM148472 & HM148717 \\
\hline C. versiforme & СРC 19053 & KT600417 & KT600515 & KT600613 \\
\hline C. xantochromaticum & CBS 140691 & LN834415 & LN834511 & LN834599 \\
\hline C. xylophilum & CBS 125997 & HM148230 & HM148476 & HM148721 \\
\hline
\end{tabular}

Table 3. GenBank accession numbers and culture collection numbers of the nucleotide sequences of the Pestalotiopsis taxa used in this study. The new sequences generated in this study are in black bold type.

\begin{tabular}{lllll}
\hline \multirow{2}{*}{ Species } & \multirow{2}{*}{ Culture Collection Number } & \multicolumn{3}{c}{ GenBank Accession Numbers } \\
\cline { 3 - 5 } & & ITS & TUB2 & TEF1 \\
\hline Neopestalotiopsis clavispora & CBS 447.73 & KM199374 & KM199443 & KM199539 \\
N. formicarum & CBS 362.72 & KM199358 & KM199455 & KM199517 \\
Pestalotiopsis adusta & MFLUCC 10-0146 & JX399006 & JX399037 & JX399070 \\
P. aggestorum & LC8186 & KY464140 & KY464160 & KY464150 \\
P. anacardiacearum & IFRDCC 2397 & KC247154 & KC247155 & KC247156 \\
P. arceuthobii & CBS 434.65 & KM199341 & KM199427 & KM199516 \\
P. arengae & CBS 331.92 & KM199340 & KM199426 & KM199515 \\
\hline
\end{tabular}


Table 3. Cont.

\begin{tabular}{|c|c|c|c|c|}
\hline \multirow{2}{*}{ Species } & \multirow{2}{*}{ Culture Collection Number } & \multicolumn{3}{|c|}{ GenBank Accession Numbers } \\
\hline & & ITS & TUB2 & TEF1 \\
\hline P. australasiae & CBS 114126 & KM199297 & KM199409 & KM199499 \\
\hline P. australis & CBS 114193 & KM199332 & KM199383 & KM199475 \\
\hline P. biciliata & CBS 124463 & KM199308 & KM199399 & KM199505 \\
\hline P. biciliata & CBS 790.68 & KM199305 & KM199400 & KM199507 \\
\hline P. biciliata & MFLUCC 12-0598 & KX816920 & KX816948 & KX816890 \\
\hline P. brachiata & LC2988 & KX894933 & KX895265 & KX895150 \\
\hline P. brassicae & CBS 170.26 & KM199379 & - & KM199558 \\
\hline P. camelliae & MFLUCC 12-0277 & JX399010 & JX399041 & JX399074 \\
\hline P. chamaeropis & CBS 186.71 & KM199326 & KM199391 & KM199473 \\
\hline P. clavata & MFLUCC 12-0268 & JX398990 & JX399025 & JX399056 \\
\hline P. colombiensis & CBS 118553 & KM199307 & KM199421 & KM199488 \\
\hline P. digitalis & ICMP 5434 & KP781879 & KP781883 & - \\
\hline P. dilucida & LC3232 & KX894961 & KX895293 & KX895178 \\
\hline P. diploclisiae & CBS 115587 & KM199320 & KM199419 & KM199486 \\
\hline P. distincta & LC8185 & KY464139 & KY464159 & KY464149 \\
\hline P. diversiseta & MFLUCC 12-0287 & JX399009 & JX399040 & JX399073 \\
\hline P. dracontomelon & MFUCC 10-0149 & KР781877 & - & KP781880 \\
\hline P. ericacearum & IFRDCC 2439 & KC537807 & KC537821 & KC537814 \\
\hline P. ericacearum & OP023 & KC537807 & KC537821 & KC537814 \\
\hline P. formosana & NTUCC 17-010 & MH809382 & MH809386 & MH809390 \\
\hline P. furcata & MFLUCC 12-0054 & JQ683724 & JQ683708 & JQ683740 \\
\hline P. gaultheria & IFRD 411-014 & КС537805 & КС537819 & КС537812 \\
\hline P. gaultheria & OP137 & КС537805 & КС537819 & KC537812 \\
\hline P. grevilleae & CBS 114127 & KM199300 & KM199407 & KM199504 \\
\hline P. hawaiiensis & CBS 114491 & KM199339 & KM199428 & KM199514 \\
\hline P. hispanica & CBS 115391 & MH553981 & MH554640 & MH554399 \\
\hline P. hollandica & CBS 265.33 & KM199328 & KM199388 & KM199481 \\
\hline P. humus & CBS 336.97 & KM199317 & KM199420 & KM199484 \\
\hline P. inflexa & MFLUCC 12-0270 & JX399008 & JX399039 & JX399072 \\
\hline P. intermedia & MFLUCC 12-0259 & JX398993 & JX399028 & JX399059 \\
\hline P. italiana & MFLUCC 12-0657 & KP781878 & KP781882 & KP781881 \\
\hline P. jesteri & CBS109350 & KM199380 & KM199468 & KM199554 \\
\hline P. jiangxiensis & LC4399 & KX895009 & KX895341 & KX895227 \\
\hline P. jinchanghensis & LC8191 & KY464145 & KY464165 & KY464155 \\
\hline P. kenyana & CBS 442.67 & KM199302 & KM199395 & KM199502 \\
\hline P. knightiae & CBS 114138 & KM199310 & KM199408 & KM199497 \\
\hline P. krabiensis & MFLUCC 16-0260 & MH388360 & MH412722 & MH388395 \\
\hline P. leucadendri & CBS 121417 & MH553987 & MH554654 & MH554412 \\
\hline P. licualacola & HGUP 4057 & KC492509 & KC481683 & KC481684 \\
\hline P. linearis & MFLUCC 12-0271 & JX398992 & JX399027 & JX399058 \\
\hline P. longiappendiculata & LC3013 & KX894939 & KX895271 & KX895156 \\
\hline P. lushanensis & LC8183 & KY464137 & KY464157 & KY464147 \\
\hline P. lushanensis & OP086 & KC537804 & KC537818 & KC537811 \\
\hline P. macadamiae & BRIP 63738 & KX186588 & KX186680 & KX186621 \\
\hline P. malayana & CBS 102220 & KM199306 & KM199411 & KM199482 \\
\hline P. monochaeta & CBS 144.97 & KM199327 & KM199386 & KM199479 \\
\hline P. montelica & MFLUCC 12-0279 & JX399012 & JX399043 & JX399076 \\
\hline P. neolitseae & NTUCC 17-012 & MH809384 & MH809388 & MH809392 \\
\hline P. novae-hollandiae & CBS 130973 & KM199337 & KM199425 & KM199511 \\
\hline P. oryzae & CBS 353.69 & KM199299 & KM199398 & KM199496 \\
\hline P. pandanicola & MFLUCC 16-0255 & MH388361 & MH412723 & MH388396 \\
\hline P. papuana & CBS 331.96 & KM199321 & KM199413 & KM199491 \\
\hline P. parva & CBS 265.37 & KM199312 & KM199404 & KM199508 \\
\hline P. pinicola & KUMCC 19-0183 & MN412636 & MN417507 & MN417509 \\
\hline P. pinicola & KUMCC 19-0203 & MN412637 & MN417508 & MN417510 \\
\hline
\end{tabular}


Table 3. Cont.

\begin{tabular}{lllll}
\hline \multirow{2}{*}{ Species } & \multirow{2}{*}{ Culture Collection Number } & \multicolumn{2}{c}{ GenBank Accession Numbers } \\
\cline { 3 - 5 } & & ITS & TUB2 & TEF1 \\
\hline P. portugalica & CBS 393.48 & KM199335 & KM199422 & KM199510 \\
P. rhododendri & IFRDCC 2399 & KC537804 & KC537818 & KC537811 \\
P. rhodomyrtus & HGUP 4230 & KF412648 & KC537818 & KF412645 \\
P. rosea & CL0441 & KY228790 & - & - \\
P. rosea & MFLUCC 12-0258 & JX399005 & JX399036 & JX399069 \\
P. scoparia & CBS 176.25 & KM199330 & KM199393 & KM199478 \\
P. sequoia & MFLUCC 13-0399 & KX572339 & - & - \\
P. shorea & MFLUCC 12-0314 & KJ503811 & KJ503814 & KJ503817 \\
P. spathulata & CBS 356.86 & KM199338 & KM199423 & KM199513 \\
P. spathuliappendiculata & CBS 144035 & MH554172 & MH554845 & MH554607 \\
P. telopeae & CBS 114161 & KM199296 & KM199403 & KM199500 \\
P. terricola & CBS 141.69 & MH554004 & MH554680 & MH554438 \\
P. trachicarpicola & IFRDCC 2240 & NR_120109 & - & - \\
P. trachicarpicola & OP143 & JQ845947 & JQ845945 & JQ845946 \\
P. unicolor & MFLUCC 12-0276 & JX398999 & JX399030 & JX399063 \\
P. verruculosa & MFLUCC 12-0274 & JX398996 & - & JX399061 \\
P. yanglingensis & LC3375 & KX894975 & KX895307 & KX895192 \\
\hline
\end{tabular}

\section{Results}

\subsection{Phylogenetic Analysis of Combined Sequence Data}

The combined dataset of genera Cladosporium and Pestalotiopsis were analyzed using maximum likelihood and Bayesian analyses (Figure 2; Figure 4). Both the ML and BYPP trees showed similar results in topology and no significant differences were seen (data not presented).

In the Cladosporium tree (Figure 2), the final alignments contained 104 strains with 1484 characters, including 594 characters for TEF1, 306 characters for ACT, and 584 characters for ITS. Cercospora beticola (CBS 116456) was used as an outgroup taxon. The tree topology of the ML analysis was similar to the BYPP. The best scoring RAxML tree with a final likelihood value of $-14,457.527098$ is presented. The matrix had 681 distinct alignment patterns with $30.20 \%$ undetermined characters or gaps. Estimated base frequencies were as follows: $A=0.228336, C=0.290122, G=0.251877, T=0.229664$; substitution rates $\mathrm{AC}=1.724785, \mathrm{AG}=2.866615, \mathrm{AT}=1.692026, \mathrm{CG}=1.001444, \mathrm{CT}=5.300862, \mathrm{GT}=1.000000$; gamma distribution shape parameter $\mathrm{a}=0.312597$. The phylogram of the genus Cladosporium based on a combined dataset showed that our strains grouped together with Cladosporium anthropophilum clade with relatively high bootstrap supports (Figure 2).

In the Pestalotiopsis tree (Figure 4), the final alignments contained 81 strains with 1562 characters, including 549 characters for TEF1, 440 characters for TUB2, and 573 characters for ITS. Neopestalotiopsis formicarum (CBS 362.72) and N. clavispora (CBS 447.73) were used as outgroup taxa. The tree topology of the ML analysis was similar to the BYPP. The best scoring RAxML tree with a final likelihood value of -11413.131729 is presented. The matrix had 696 distinct alignment patterns, with $12.40 \%$ undetermined characters or gaps. Estimated base frequencies were as follows: $A=0.235816, C=0.293897, G=0.211788$, $\mathrm{T}=0.258500 ;$ substitution rates $\mathrm{AC}=1.049115, \mathrm{AG}=3.327441, \mathrm{AT}=1.067008, \mathrm{CG}=0.861291$, $\mathrm{CT}=3.485808, \mathrm{GT}=1.000000$; gamma distribution shape parameter $\mathrm{a}=0.276615$. The Pestalotiopsis phylogram, based on a combined dataset, showed that our new species, Pestalotiopsis pinicola, was well separated from $P$. rosea with relatively high bootstrap supports (100\% ML/1 BYPP, Figure 4). Therefore, we propose Pestalotiopsis pinicola as a distinct new species and Cladosporium anthropophilum as a previously known species. 


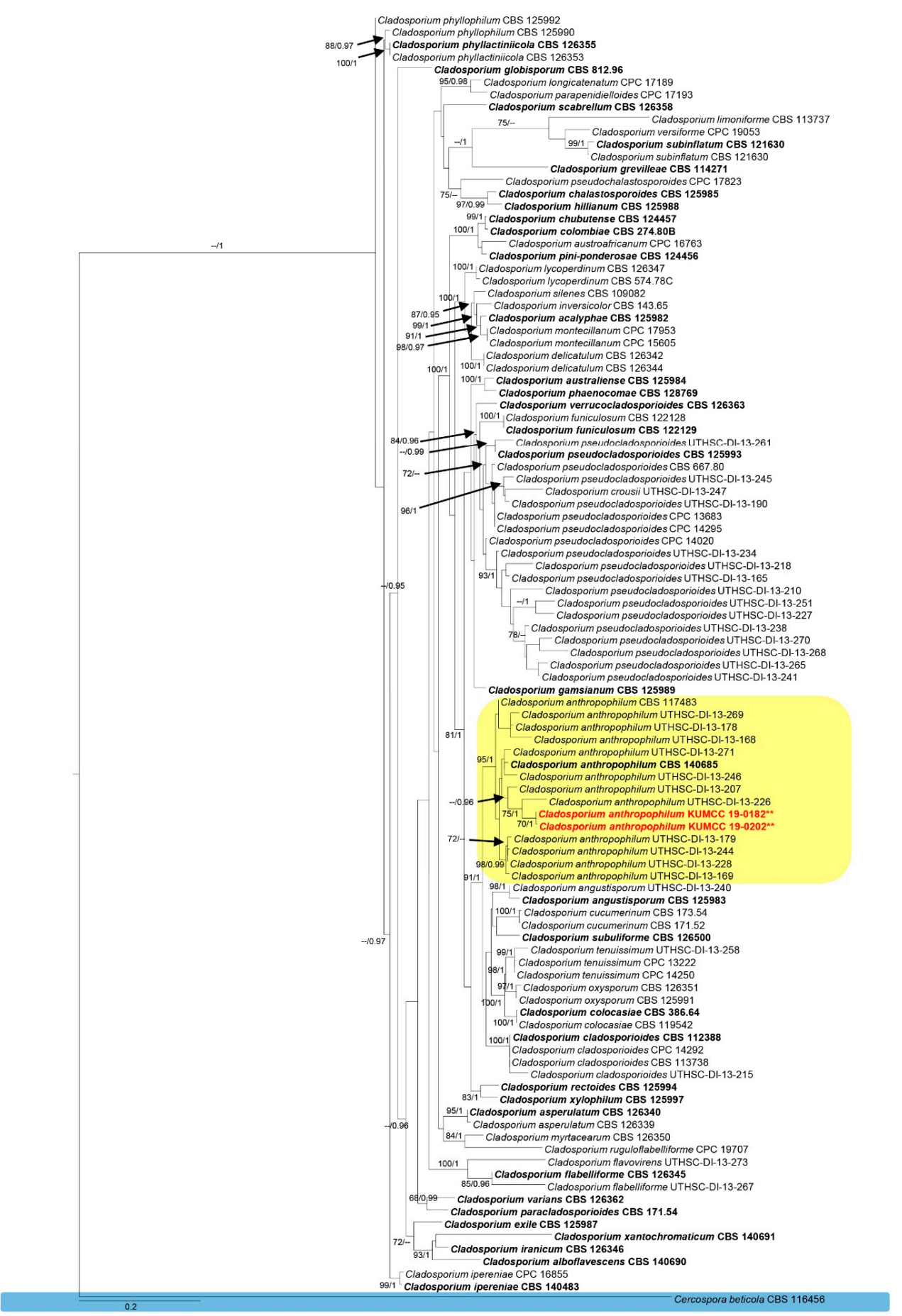

Figure 2. Phylogram generated from RAxML analysis based on combined TEF1, ACT and ITS sequence data of the genus Cladosporium. Related sequences were obtained from Sandoval-Denis et al. [16] and Bensch et al. [56]. Bootstrap support values for ML equal to or greater than $60 \%$ and BYPP from MCMC analyses equal to or greater than 0.95 are given above/below the nodes. The ex-type strains are indicated in bold type. Newly generated sequences are indicated in red with two asterisks.

\subsection{Taxonomy}

Cladosporium anthropophilum Sand.-Den., Gené and Wiederhold, Persoonia 36: 290 (2016) [16]. Index Fungorum number: IF815334, Facesoffungi number: FoF 06275, Figure 3.

Saprobic or weak pathogen on seed coat of Pinus armandii. Sexual morph: Undetermined. Asexual morph: Mycelium sparsely formed, superficial, overgrowing entire pod, thin to dense, later often 
forming colonies on the surface, hyphae straight to strongly flexuous-sinuous, branched, subhyaline to olivaceous-brown. Conidiophores erect, stipes, slightly attenuated towards the apex, yellow-brown to dark-brown, smooth and thick-walled, branched, septate. Conidiogenous cells 5-15 × 2.5-5.5 $\mu \mathrm{m}$ $(\bar{x}=8.7 \times 4 \mu \mathrm{m} ; n=20)$, cylindrical, sometimes geniculate-sinuous, proliferation sympodia with distinctive scar. Secondary ramoconidia 5.9-9.1 $\times 2-3.5 \mu \mathrm{m}(\overline{\mathrm{x}}=7.7 \times 2.9 \mu \mathrm{m} ; n=40)$, olivaceous-brown, ellipsoid-ovoid, obovoid, fusiform, subcylindrical, aseptate, smooth to rough-walled, granulate and scars. Conidia $2.7-5.6 \times 2-3.2 \mu \mathrm{m}(\overline{\mathrm{x}}=4.1 \times 2.7 \mu \mathrm{m} ; n=40)$, in simple or branched chains, subhyaline to olivaceous, ellipsoid-ovoid, aseptate, a scar at base, rough-walled with granulate.
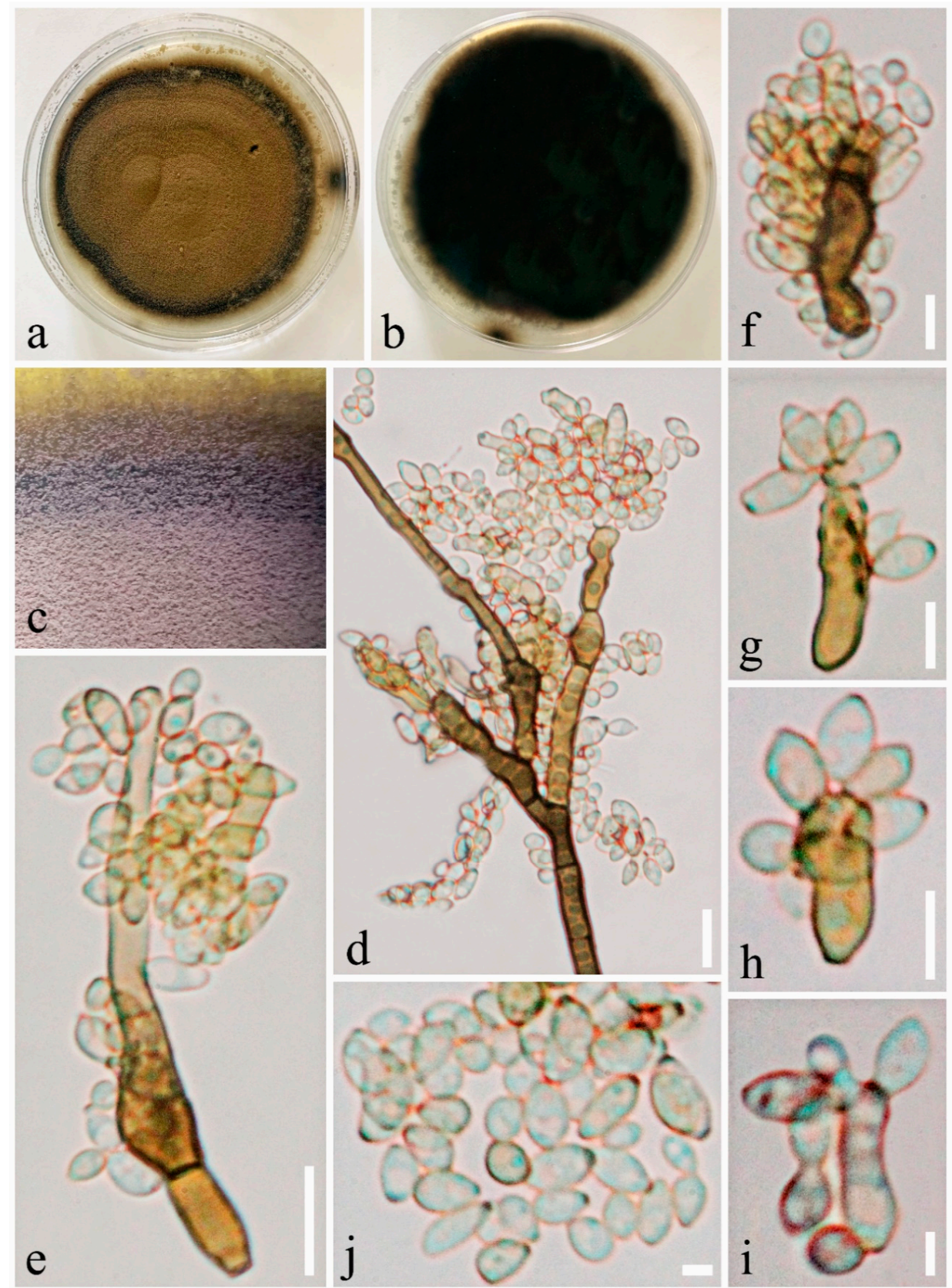

Figure 3. Cladosporium anthropophilum (KUMCC 19-0182). (a,b) Colony on PDA media. (c) Mycelium masses. (d,e) Conidiophores and conidiogenous cells and conidia. (f-i) Conidiogenous cells with secondary ramoconidia and coidia. (j) Conidia. Scale bars: $(\mathbf{d}, \mathbf{e})=10 \mu \mathrm{m},(\mathbf{f}-\mathbf{i})=5 \mu \mathrm{m},(\mathbf{j})=2 \mu \mathrm{m}$.

Culture characters: Colonies on PDA reaching $9 \mathrm{~cm}$ in diameter after 3 weeks at room temperature. Colonies olivaceous-grey to olivaceous, pale-olivaceous to black at the margin and circular with slightly 
regular colony, powdery, radially furrowed, aerial mycelium sparse with raised elevation, numerous small prominent exudates formed, sporulation profuse.

Material examined: CHINA, Yunnan Province, on seed coat of Pinus armandii Franch., May 2019, Kai Yan, Seed01 (MFLU19-2362); living culture KUMCC 19-0182 = KUMCC 19-0202.

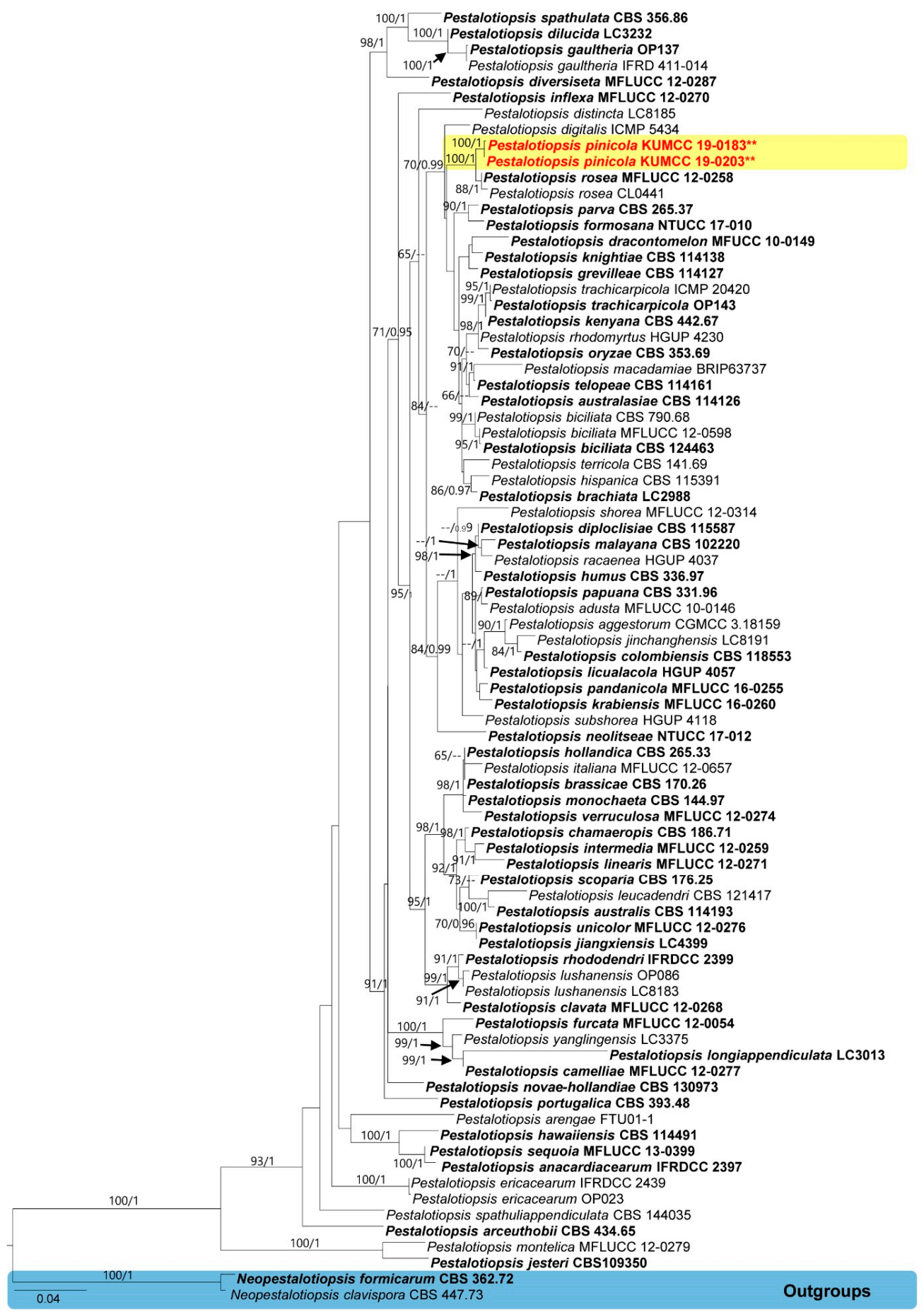

Figure 4. Phylogram generated from RAxML analysis based on combined TEF1, TUB2, and ITS sequence data of the genus Pestalotiopsis. Related sequences were obtained from Ariyawansa et al. [55] and Tibpromma et al. $[9,18]$. Bootstrap support values for ML equal to or greater than $60 \%$ and BYPP from MCMC analyses equal to or greater than 0.95 are given above/below the nodes. The ex-type strains are indicated in bold type. Newly generated sequences are indicated in red with two asterisks $\left.{ }^{* *}\right)$. 
Note that Cladosporium anthropophilum was established by Sandoval-Denis et al. [16] which belongs to the C. cladosporioides species complex. Cladosporium anthropophilum is probably known as a common saprobic fungus and also represents a clinically relevant fungus [16,70]. In this study, we found a strain of C. anthropophilum from a seed coat of Pinus armandii which was confirmed based on morphology and multi-gene analysis (Figures 2 and 3). The morphology of our strain was similar to the C. anthropophilum described by Sandoval-Denis et al. [16]. In addition, this is the first report of C. anthropophilum from P. armandii (Figure 4).

Pestalotiopsis pinicola Tibpromma, Karunaratha and Mortimer, sp. nov.

Index Fungorum number: IF556765, Facesoffungi number: FoF 06276, Figure 5.

Etymology: named after the host genus, Pinus.

Holotype: MFLU19-2363.

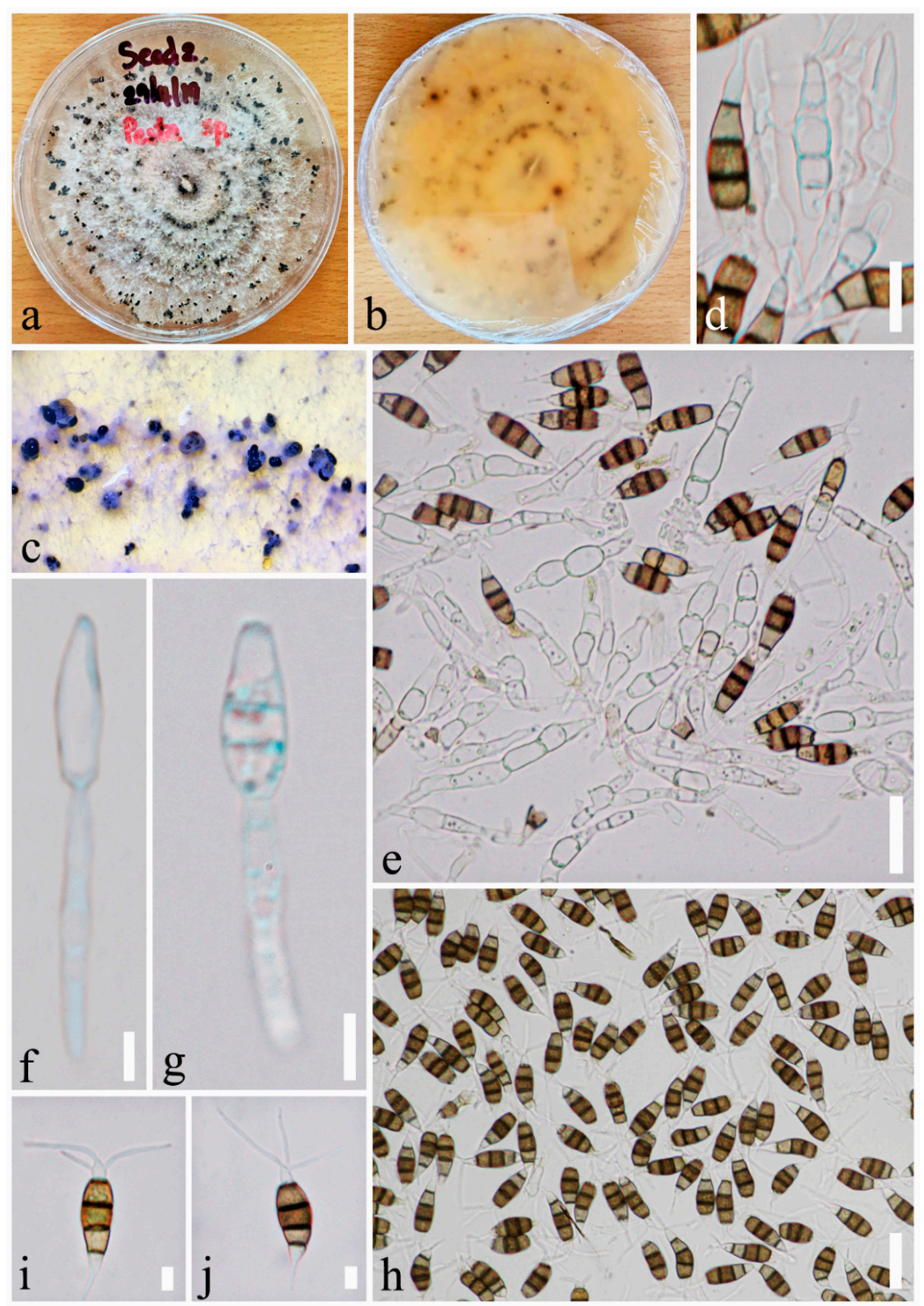

Figure 5. Pestalotiopsis pinicola (KUMCC 19-0183, ex-type). (a,b) Colony on PDA media. (c) Fruiting body on PDA media. ( $\mathbf{d}-\mathbf{g})$ Conidia, conidiogenous cells and conidia. (h-j) Conidia. Scale bars: $(\mathbf{d})=5 \mu \mathrm{m},(\mathbf{e})=10 \mu \mathrm{m},(\mathbf{f}, \mathbf{g})=5 \mu \mathrm{m},(\mathbf{h})=20 \mu \mathrm{m},(\mathbf{i}-\mathbf{j})=5 \mu \mathrm{m}$. 
Saprobic or endophytic on seed endosperm of Pinus armandii. Sexual morph: Undetermined. Asexual morph: Conidiophores short, unbranched, reduced to conidiogenous cells. Conidiogenous cells discrete, holoblastic, simple, filiform, smooth and thin-walled, hyaline. Conidia fusoid to ellipsoid, straight to slightly curved, 3-4 septate (mostly 4 septate), $18-23 \times 5-7 \mu \mathrm{m}(\overline{\mathrm{x}}=21 \times 6 \mu \mathrm{m}, n=40)$, basal cell conic to obconic with obtuse end, subhyaline, thin-walled, verruculose, $3.5-5 \mu \mathrm{m}$ long $(\bar{x}=4 \mu \mathrm{m})$; three median cells, doliiform, yellow-brown and becoming brown with age, septa and periclinal walls darker than rest of the cell, together 11-16 $\mu \mathrm{m}$ long $(\overline{\mathrm{x}}=13 \mu \mathrm{m})$; second cell from base 3-6 $\mu \mathrm{m}$ long $(\overline{\mathrm{x}}=4.5 \mu \mathrm{m})$; third cell 3-5.5 $\mu \mathrm{m}$ long $(\overline{\mathrm{x}}=4.6 \mu \mathrm{m})$; fourth cell 3-5 $\mu \mathrm{m}$ long $(\overline{\mathrm{x}}=3.9 \mu \mathrm{m})$; apical cell hyaline, conic $3-5 \mu \mathrm{m}$ long $(\overline{\mathrm{x}}=3.9 \mu \mathrm{m})$, with $2(-3)$ tubular apical appendages; appendages arising from the apex of the apical cell, unbranched, $5-17 \mu \mathrm{m}$ long $(\overline{\mathrm{x}}=10.3 \mu \mathrm{m})$; single basal appendage usually present, $2-7 \mu \mathrm{m}$ long $(\overline{\mathrm{x}}=4.7 \mu \mathrm{m})$, tubular, unbranched, centric.

Culture characteristics: Colonies on PDA reaching $9 \mathrm{~cm}$ in diameter after 2 weeks at room temperature, edge undulate with curled, whitish, aerial mycelium on surface, spore masses form after 1 month, black spore masses; reverse of culture yellow-white to yellow-orange with black dots.

Material examined: China, Yunnan Province, on endosperm of pine seed of Pinus armandii Franch., May 2019, Kai Yan, Seed02 (MFLU19-2363, holotype); ex-type living culture KUMCC 19-0183 = KUMCC 19-0203.

Note that Pestalotiopsis pinicola is introduced based on morphological and phylogenetic data. In the phylogenetic analysis, our new species cluster with P. rosea Maharachch. and K.D. Hyde [40] but are well separated with high support (100\% ML/1 BYPP, Figure 4). In addition, base pair differences of our new taxa with closest taxa were checked based on the recommendations of Jeewon and Hyde [71]; our isolate differs from P. rosea (MFLUCC12-0258 and CL0441) with five ITS base pairs (2.65\%), four TUB base pairs $(1.64 \%)$, and ten RPB2 base pairs (4.65\%). In addition, the culture of P. rosea was seen as a reddish colony [40], while our new species produces a whitish colony.

In a BLASTn search on the NCBI GenBank, the closest ITS sequence match of KUMCC 19-0183 is Pestalotiopsis sp. with a 99.31\% identity to the strain JSM 06261592 (KY086253), KUMCC 19-0203 is P. neglecta with $99.82 \%$ identity to the strain CBS 357.71 (MH860161.1), the closest TEF1 sequence matches of KUMCC 19-0183 and KUMCC 19-0203 were with the P. rosea strain MFLUCC12-0258 with 98.72\% (JX399069), while the closest matches with the TUB2 sequence were with the $99.53 \%$ identical P. olivacea strain PSHI2002 (DQ787834) by KUMCC 19-0183 and 99.53\% identical P. vismiae strain Q15DY (EF055259) by KUMCC 19-0203.

\section{Discussion}

In this paper, we describe a novel taxon belonging to Pestalotiopsis and a new host record of Cladosporium isolated from seeds of Pinus armandii obtained from Yunnan Province, China. Mature agar colonies sporulated in cultures with masses of conidia.

We isolated a new Pestalotiopsis species from mycelia-covered endosperms of pine seeds. Past research has yielded new species from Pestalotiopsis with similar origins; for example, several endophytic Pestalotiopsis species were isolated from the bark and needles of Pinus armandii in China [38]. Furthermore, Pestalotiopsis brassicae and P. oryzae were isolated from seeds from Oryza sativa and Brassica napus [42]. Several have often been isolated as endophytes and many pathogens or endophytes may persist as saprobes, which mean Pestalotiopsis species are able to switch life-modes [42]. The present study illustrates a novel species of Pestalotiopsis as Pestalotiopsis pinicola, taking both morphology and phylogeny into consideration (Figures 4 and 5). The phylogenetic tree construction of the DNA sequences of single and combined genes provides confirmation with high bootstrap support that P. pinicola is a characteristic new species separate from other known species of the genus (Figure 4). Moreover, this genus is known as one of the fungal groups that can produce a wide range of chemically novel secondary metabolites and mycotoxins; for example, pestaloside exhibiting significant antifungal properties was produced by P. microspora, obtained from Torreya taxifolia [42,72-74]. There is, consequently, a potential health threat in the sale of these seeds as an edible foodstuff. Follow-up research investigating the potential 
toxins produced by P. pinicola should be conducted to clarify this issue. We conclude that fungi live inside seeds as endophytes and then switch life-modes to saprobes or weak pathogens when conditions become unfavorable. In the future, knowledge about pestalotioid fungi associated with seeds will help provide a basis for developing proper management of these pathogens.

We found another species, Cladosporium anthropophilum, growing on pine seed coats. The etymology of this species comes from Greek which refers to the sample's source which was isolated from a human clinical sample [16]. This species can be found in human clinical samples, indoor air, food and plant materials, such as seeds or leaves, and it is also a common saprobic fungus [56]. In addition, this species is known as the second-most prevalent species from clinical environments from the US after C. halotolerans, and it also has been isolated quite frequently from indoor environments $[16,69]$. However, we continue to lack information about the chemistry or secondary metabolites of this species along with the potential serious health effects associated with long-term exposure to a large amount of Cladosporium anthropophilum.

The present study illustrates two species of Pestalotiopsis and Cladosporium based on both morphology and phylogeny. These two species of fungi were isolated from pine seeds from Yunnan Province, China. The fungal mycelia in the seeds were observed after the seeds were broken open to eat, and these seeds can be found in many food markets around Yunnan Province. We recommend that consumers should carefully check seed products before purchase and consumption, as these fungi may cause adverse health effects in the long term. Therefore, to address this health concern, in the future we will focus our research on the secondary metabolites and mycotoxins of Cladosporium anthropophilum and Pestalotiopsis pinicola.

Author Contributions: S.T., P.E.M., S.C.K. and K.Y. designed the experiments. S.T. and S.C.K. conducted the experiments. S.T. performed the morphological study and phylogenetic data. S.T., P.E.M., K.Y., and S.C.K. analyzed the data. S.T., P.E.M., I.P., F.Z., K.Y., and S.C.K. provided funding and financial support for this study. S.T. wrote the manuscript and P.E.M., F.Z., J.X., S.C.K., I.P., and K.Y. gave comments, suggestions, and edited the manuscript. All authors reviewed and approved the final manuscript.

Funding: This research was funded by National Science Foundation of China (NSFC) [project codes 41761144055, 31750110478, 41807524 and 41771063], the Southeast Asian Biodiversity Research Institute [project code Y4ZK111B01] and Chiang Mai University.

Acknowledgments: S.T. would like to thank the International Postdoctoral Exchange Fellowship Program (number Y9180822S1), CAS President's International Fellowship Initiative (PIFI) (number 2020PC0009), China Postdoctoral Science Foundation, and the Yunnan Human Resources and Social Security Department Foundation for funding her postdoctoral research. Key Laboratory for Plant Diversity and Biogeography of East Asia, Kunming Institute of Botany, Chinese Academy of Science, Kunming, China and Gu Zhi-Jia are thanked for the scanning electron microscopy morphology. P.E.M. thanks the National Science Foundation of China (NSFC), project codes 41761144055 and 41771063, and the Southeast Asian Biodiversity Research Institute (Y4ZK111B01) for support. S.C.K. thanks CAS President's International Fellowship Initiative (PIFI) for funding his postdoctoral research (number 2018PC0006) and the National Science Foundation of China (NSFC) for funding this research work under project code 31750110478. This research was partially supported by the Chinese National Science Foundation (41807524) and Chiang Mai University. Austin Smith at World Agroforestry (ICRAF), Kunming Institute of Botany, China, is thanked for English editing.

Conflicts of Interest: There is no conflict of interest.

\section{References}

1. Anon. Dendrology (Southern Edition); China Forest Press: Beijing, China, 1994. (In Chinese)

2. Yan, K.; He, X.Y.; Wei, C.; Tao, L.; Zhang, G. Variation of Antioxidant System in Pinus armandii under Elevated O3 in an Entire Growth Season. Acta Hydrochim. Hydrobiol. 2013, 41, 5-10.

3. Destaillats, F.; Cruzhernandez, C.; Giuffrida, F.; Dionisi, F. Identification of the botanical origin of pine nuts found in food products by gas-liquid chromatography analysis of fatty acid profile. J. Agric. Food Chem. 2010, 58, 2082-2087. [CrossRef] [PubMed]

4. CCCFNA. Chinese pine nuts. In China Chamber of Commerce of Imports/Exports of Foodstuffs, Native Produce, and Animal by-Products; The International Tea Committee, International Nut and Dried Fruit Council: Reus, Spain, 2011. 
5. Joshi, V.; Mukerji, K.G. Seed-borne mycoflora of two under-exploited legumes: Vigna umbellata and psophocarpus tetragonolobus from Northeastern parts of India. In From Ethnomycology to Fungal Biotechnology; Springer: Boston, MA, USA, 1999; pp. 257-268.

6. Cram, M.M.; Fraedrich, S.W. Seed diseases and seedborne pathogens of North America. Tree Plant. Notes. 2010, 53, 35-44.

7. Sutherland, J.R.; Miller, T.; Quinard, R.S. Cone and seed diseases of North American conifers. N. Am. For. Comm. Publ. Number 1 1987, 1, 1-70.

8. Promputtha, I.; Lumyong, S.; Dhanasekaran, V.; Mckenzie, E.H.C.; Hyde, K.D.; Jeewon, R. A phylogenetic evaluation of whether endophytes become saprotrophs at host senescence. Microb. Ecol. 2007, 53, 579-590. [CrossRef]

9. Tibpromma, S.; Hyde, K.D.; Bhat, J.D.; Mortimer, P.E.; Xu, J.C.; Promputtha, I.; Doilom, M.; Yang, J.-B.; Tang, A.M.C.; Karunarathna, S.C. Identification of endophytic fungi from leaves of Pandanaceae based on their morphotypes and DNA sequence data from southern Thailand. MycoKeys 2018, 33, 25-67. [CrossRef]

10. Mittal, R.K.; Anderson, R.L.; Mathur, S.B. Microorganisms associated with tree seeds: World checklist Petawawa National Forestry Institute, Chalk River, Ontario. Available online: https://cfs.nrcan.gc.ca/ publications?id=10573 (accessed on 29 November 2019).

11. Pittayakhajonwut, P.; Theerasilp, M.; Kongsaeree, P.; Rungrod, A.; Tanticharoen, M.; Thebtaranonth, Y.; Pughiinin, A. A sesquiterpene from the fungus Kionochaeta pughii BCC 3878. Planta Med. 2002, 68, 1017-1019. [CrossRef]

12. Chinworrungsee, M.; Kittakoop, P.; Isaka, M.; Maithip, P.; Supothina, S.; Thebtaranonth, Y. Isolation and structure elucidation of a novel antimalarial macrocyclic polylactone, menisporopsin A, from the fungus Menisporopsis theobromae. J. Nat. Prod. 2004, 67, 689-692. [CrossRef]

13. Link, H.F. Observationes in ordines plantarum naturales. Observationes in ordines plantarum naturales. 2. Mag. Gesell. Naturf. Freunde Berlin 1816, 8, 25-45.

14. Bensch, K.; Braun, U.; Groenewald, J.Z.; Crous, P.W. The genus Cladosporium. Stud. Mycol. 2012, 72, 1-401. [CrossRef]

15. Crous, P.W.; Shivas, R.G.; Quaedvlieg, W.; van der Bank, M.; Zhang, Y.; Summerell, B.A.; Guarro, J.; Wingfield, M.J.; Wood, A.R.; Alfenas, A.C.; et al. Fungal planet description sheets: 214-280. Persoonia 2013, 31, 188. [CrossRef] [PubMed]

16. Sandoval-Denis, M.; Genã, J.; Sutton, D.A.; Wiederhold, N.P.; Cano-Lira, J.F.; Guarro, J. New species of Cladosporium associated with human and animal infections. Persoonia 2016, 36, 281-298. [CrossRef] [PubMed]

17. Ma, R.; Chen, Q.; Fan, Y.; Wang, Q.; Chen, S.F.; Liu, X.Z.; Cai, L.; Yao, B. Six new soil-inhabiting Cladosporium species from plateaus in China. Mycologia 2017, 109, 1-17. [CrossRef] [PubMed]

18. Tibpromma, S.; Hyde, K.D.; Mckenzie, E.H.C.; Bhat, D.J.; Phillips, A.J.L.; Wanasinghe, D.N.; Samarakoon, M.C.; Jayawardena, R.S.; Dissanayake, A.J.; Tennakoon, D.S.; et al. Fungal diversity notes 840-928: Micro-fungi associated with Pandanaceae. Fungal Divers. 2018, 72, 1-160. [CrossRef]

19. Fradkin, A.; Tarlo, S.M.; Tobin, R.S.; Tucic-Porretta, M.; Malloch, D. Species identification of airborne molds and its significance for the detection of indoor pollution. Air Repair. 1987, 37, 51-53. [CrossRef]

20. Flannigan, B.; Samson, R.A.; Miller, J.D. Microorganisms in Home and Indoor Work Environments; CRC Press: Boca Raton, FL, USA, 2011; Volume 3, pp. 55-58.

21. Bullerman, L.B. SPOILAGE|Fungi in food-An overview. Encycl. Food Sci. Nutr. 2003, 1, 5511-5522.

22. Horner, W.E.; Worthan, A.G.; Morey, P.R. Air-and dustborne mycoflora in houses free of water damage and fungal growth. Appl. Environ. Microbiol. 2004, 70, 6394-6400. [CrossRef]

23. Horner, W.E.; Helbling, A.; Salvaggio, J.E.; Lehrer, S.B. Fungal allergens. Clin. Microbiol. Rev. 1995, 8, 161-179. [CrossRef]

24. Sundell, J. On the history of indoor air quality and health. Indoor Air. 2004, 14, 8-51. [CrossRef]

25. Matheson, M.C.; Abramson, M.J.; Dharmage, S.C.; Forbes, A.B.; Raven, J.M.; Thien, F.C.; Walters, E.H. Changes in indoor allergen and fungal levels predict changes in asthma activity among young adults. Clin. Exp. Allergy 2010, 35, 907-913. [CrossRef] [PubMed]

26. Denning, D.W.; O'Driscoll, B.R.; Hogaboam, C.M.; Bowyer, P.; Niven, R.M. The link between fungi and severe asthma: A summary of the evidence. Eur. Respir. J. 2006, 27, 615-626. [CrossRef] [PubMed]

27. Simonnobbe, B.; Denk, U.; Pöll, V.; Rid, R.; Breitenbach, M. The spectrum of fungal allergy. Int. Arch. Allergy Immunol. 2008, 145, 58-86. [CrossRef] [PubMed] 
28. Mari, A.; Rasi, C.; Palazzo, P.; Scala, E. Allergen databases: Current status and perspectives. Curr. Allergy Asthma Rep. 2009, 9, 376-384. [CrossRef] [PubMed]

29. Reboux, G.; Bellanger, A.P.; Roussel, S.; Grenouillet, F.; Millon, L. Moulds in dwellings: Health risks and involved species. Rev. Mal. Respir. 2010, 27, 169-179. [CrossRef] [PubMed]

30. Heinzerling, L.; Mari, A.; Bergmann, K.C.; Bresciani, M.; Burbach, G.; Darsow, U.; Durham, S.; Fokkens, W.; Gjomarkaj, M.; Haahtela, T.; et al. The skin prick test-European standards. Clin. Transl. Allergy 2013, 3, 1-10. [CrossRef]

31. Segers, F.J.J.; Meijer, M.; Houbraken, J.; Samson, R.A.; Han, A.B.W.; Dijksterhuis, J. Xerotolerant Cladosporium sphaerospermum are predominant on indoor surfaces compared to other Cladosporium species. PLoS ONE 2015, 10, 145-150. [CrossRef]

32. Jayasiri, S.C.; Hyde, K.D.; Jones, E.B.G.; McKenzie, E.H.C.; Jeewon, R.; Phillips, A.J.L.; Bhat, D.J.; Wanasinghe, D.N.; Liu, J.K.; Lu, Y.Z.; et al. Diversity, morphology and molecular phylogeny of Dothideomycetes on decaying wild seed pods and fruits. Mycosphere 2019, 10, 1-186. [CrossRef]

33. Chen, M.M. Forest Fungi Phytogeography: Forest Fungi Phytogeography of China, North America, and Siberia and International Quarantine of Tree Pathogens; Pacific Mushroom Research and Education Center: Sacramento, CA, USA, 2002.

34. Farr, D.F.; Rossman, A.Y. Fungal Databases, US National Fungus Collections, ARS, USDA. Available online: https://nt.ars--grin.gov/fungaldatabases (accessed on 1 August 2019).

35. Steyaert, R.L. Contribution a l'etude monographique de Pestalotia de Not. et Monochaetia Sacc. (Truncatella gen. nov. et Pestalotiopsis gen. nov.). Available online: https://www.jstor.org/stable/3666710?seq=1 (accessed on 1 August 2019).

36. Jeewon, R.; Liew, E.C.Y.; Hyde, K.D. Phylogenetic relationships of Pestalotiopsis and allied genera inferred from ribosomal DNA sequences and morphological characters. Mol. Phylogenet. Evol. 2002, 25, 378-392. [CrossRef]

37. Jeewon, R.; Liew, E.C.Y.; Simpson, J.A.; Hodgkiss, I.J.; Hyde, K.D. Phylogenetic significance of morphological characters in the taxonomy of Pestalotiopsis species. Mol. Phylogenet. Evol. 2013, 27, 372-383. [CrossRef]

38. Hu, H.; Jeewon, R.; Zhou, D.; Zhou, T.; Hyde, K.D. Phylogenetic diversity of endophytic Pestalotiopsis species in Pinus armandii and Ribes spp.: Evidence from rDNA and $\beta$-tubulin gene phylogenies. Fungal Divers. 2007, 45-89.

39. Maharachchikumbura, S.S.N.; Guo, L.D.; Chukeatirote, E.; Bahkali, A.H.; Hyde, K.D. Pestalotiopsis-morphology, phylogeny, biochemistry and diversity. Fungal Divers. 2011, 50, 167-187. [CrossRef]

40. Maharachchikumbura, S.S.N.; Guo, L.D.; Chukeatirote, E.; Wu, W.P.; Sun, X.; Crous, P.W.; Bhat, D.J.; McKenzie, E.H.C.; Bahkali, A.H.; Hyde, H.D. A multi-locus backbone tree for Pestalotiopsis, with a polyphasic characterization of 14 new species. Fungal Divers. 2012, 56, 95-129. [CrossRef]

41. Maharachchikumbura, S.S.N.; Guo, L.D.; Chukeatirote, E.; Hyde, K.D. Improving the backbone tree for the genus Pestalotiopsis; addition of P. steyaertii and P. magna sp. nov. Mycol. Prog. 2013, 13, 617-624. [CrossRef]

42. Maharachchikumbura, S.S.N.; Hyde, K.D.; Groenewald, J.Z.; Xu, J.; Crous, P.W. Pestalotiopsis revisited. Stud. Mycol. 2014, 79, 121-186. [CrossRef] [PubMed]

43. Maharachchikumbura, S.S.N.; Guo, L.D.; Liu, Z.Y.; Hyde, K.D. Pseudopestalotiopsis ignota and Ps. camelliae spp. nov. associated with grey blight disease of tea in China. Mycol. Prog. 2016, 15, 22-58. [CrossRef]

44. Maharachchikumbura, S.S.N.; Hyde, K.D.; Jones, E.B.G.; Mckenzie, E.H.C.; Bhat, J.D.; Dayarathne, M.C.; Huang, C.-K.; Norphanphoun, C.; Senanayake, I.C.; Perera, R.H.; et al. Families of Sordariomycetes. Fungal Divers. 2016, 79, 1-317. [CrossRef]

45. Sutton, D.A. Coelomycetous fungi in human disease. A review: Clinical entities, pathogenesis, identification and therapy. Rev. Iberoam. Micol. 1999, 16, 171-179.

46. Monden, Y.; Yamamoto, S.; Sunada, A.; Asari, S.; Makimura, K.; Inoue, Y. First case of fungal keratitis caused by Pestalotiopsis clavispora. Clin. Ophthalmol. 2013, 7, 2261-2264. [CrossRef]

47. Zhuang, W.Y. Fungi of Northwestern China. Mycotaxon, Ltd.: Ithaca, NY, USA, 2005.

48. Figueras, M.J.; Guarro, J. A scanning electron microscopic study of ascoma development in Chaetomium Malays. Mycologia 1988, 80, 298-306. [CrossRef]

49. Index Fungorum. Available online: http://www.indexfungorumorg/Names/Namesasp (accessed on 15 July 2019). 
50. Jayasiri, S.C.; Hyde, K.D.; Ariyawansa, H.A.; Bhat, J.; Buyck, B.; Cai, L.; Dai, Y.C.; Abd-Elsalam, K.A.; Ertz, D.; Hidayat, I.; et al. The Faces of Fungi database: Fungal names linked with morphology, phylogeny and human impacts. Fungal Divers. 2015, 74, 3-18. [CrossRef]

51. Carbone, I.; Kohn, L.M. A method for designing primer sets for speciation studies in filamentous ascomycetes. Mycologia 1999, 91, 553-556. [CrossRef]

52. White, T.J.; Bruns, T.; Lee, S.; Taylor, J. Amplification and direct sequencing of fungal ribosomal RNA genes for phylogenetics. In PCR Protocols: A Guide to Methods and Applications; Innis, M.A., Gelfand, D.H., Sninsky, J.J., White, T.J., Eds.; Academic Press: New York, NY, USA, 1990; pp. 315-322.

53. Rehner, S.A. Primers for Elongation Factor 1-alpha (EF1-alpha). 2001. Available online: http://www.aftol. org/pdfs/EF1primer.pdf (accessed on 15 July 2019).

54. O'Donnell, K.; Cigelnik, E. Two divergent intragenomic rDNA ITS2 types within a monophyletic lineage of the fungusfusariumare nonorthologous. Mol. Phylogenet. Evol. 1997, 7, 103-116. [CrossRef] [PubMed]

55. Ariyawansa, H.A.; Hyde, K.D. Additions to Pestalotiopsis in Taiwan. Mycosphere 2018, 9, 999-1013. [CrossRef]

56. Bensch, K.; Groenewald, J.Z.; Meijer, M.; Dijksterhuis, J.; Jurjevic, Z.; Houbraken, J.; Crous, P.W.; Samson, R.A. Cladosporium species in indoor environments. Stud. Mycol. 2018, 89, 177-301. [CrossRef]

57. Katoh, K.; Standley, D.M. A simple method to control over-alignment in the MAFFT multiple sequence alignment program. Bioinformatics 2016, 32, 1933-1942. Available online: http://mafft.cbrc.jp/alignment/ server/index.html (accessed on 15 September 2019). [CrossRef]

58. Hall, T. Bioedit Version 6.0.7; Department of Microbiology, North Carolina State University: Raleigh, NC, USA, 2004.

59. Glez-Peña, D.; Gómez-Blanco, D.; Reboiro-Jato, M.; Fdez-Riverola, F.; Posada, D. ALTER: Program-Oriented Format Conversion of DNA and Protein Alignments. Nucleic Acids Research. Web Server Issue. 2010. Available online: http://www.sing-group.org/ALTER/ (accessed on 15 September 2019).

60. Miller, M.A.; Pfeiffer, W.; Schwartz, T. Creating the CIPRES Science Gateway for inference of large phylogenetic trees. In Proceedings of the Gateway Computing Environments Workshop (GCE), New Orleans, LA, USA, 14 November 2010; Institute of Electrical and Electronics Engineers: New Orleans, LA, USA, 2010. Available online: http://www.phylo.org/ (accessed on 15 September 2019).

61. Stamatakis, A. RAxML-VI-HPC: Maximum likelihood-based phylogenetic analyses with thousands of taxa and mixed models. Bioinformatics 2006, 22, 2688-2690. [CrossRef]

62. Stamatakis, A. RAxML version 8: A tool for phylogenetic analysis and post-analysis of large phylogenies. Bioinformatics 2014, 30, 1312-1313. [CrossRef]

63. Nylander, J.A.A. MrModeltest 20 Program Distributed by the Author. Uppsala University, Evolutionary Biology Centre: Uppsala, Sweden, 2004.

64. Rannala, B.; Yang, Z.H. Probability distribution of molecular evolutionary trees: A new method of phylogenetic inference. J. Mol. Evol. 1996, 43, 304-311. [CrossRef]

65. Liu, J.K.; Doilom, M.; Wikee, S.; Li, Y.M.; Ariyawansha, H.; Boonmee, S.; Chomnunti, P.; Dai, D.Q.; Bhat, J.D.; Romero, A.I.; et al. Towards a natural classification of Botryosphaeriales. Fungal Divers. 2012, 57, $149-210$. [CrossRef]

66. Cai, L.; Jeewon, R.; Hyde, K.D. Phylogenetic investigations of Sordariaceae based on multiple gene sequences and morphology. Mycol. Res. 2006, 110, 137-150. [CrossRef] [PubMed]

67. Zhaxybayeva, O.; Gogarten, J.P. Bootstrap, Bayesian probability and maximum likelihood mapping: Exploring new tools for comparative genome analyses. Genomics 2002, 3, 4. [PubMed]

68. Ariyawansa, H.A.; Thambugala, K.M.; Manamgoda, D.S.; Jayawardena, R.; Camporesi, E.; Boonmee, S.; Wanasinghe, D.N.; Phookamsak, R.; Hongsanan, S.; Singtripop, C.; et al. Towards a natural classification and backbone tree for Pleosporaceae. Fungal Divers. 2015, 71, 85-139. [CrossRef]

69. Rambaut, A.; Drummond, A. FigTree: Tree figure drawing tool, version 1.2.2. Institute of Evolutionary Biology; University of Edinburgh: Edinburgh, Scotland, 2008.

70. Sandoval-Denis, M.; Sutton, D.A.; Martin-Vicente, A.; Cano-Lira, J.F.; Wiederhold, N.; Guarro, J.; Gené, J. Cladosporium species recovered from clinical samples in the United States. J. Clin. Microbiol. 2015, 53, 2990-3000. [CrossRef] [PubMed]

71. Jeewon, R.; Hyde, K.D. Establishing species boundaries and new taxa among fungi: Recommendations to resolve taxonomic ambiguities. Mycosphere 2016, 7, 1669-1677. [CrossRef] 
72. Lee, J.C.; Yang, X.S.; Schwartz, M.; Strobel, G.; Clardy, J. The relationship between an endangered North American tree and an endophytic fungus. Chem. Biol. 1995, 2, 721. [CrossRef]

73. Xu, J.; Ebada, S.S.; Proksch, P. Pestalotiopsis a highly creative genus: Chemistry and bioactivity of secondary metabolites. Fungal Divers. 2010, 44, 15-31. [CrossRef]

74. Yang, X.L.; Zhang, J.Z.; Luo, D.Q. The taxonomy, biology and chemistry of the fungal Pestalotiopsis genus. Nat. Prod. Rep. 2012, 29, 622-641. [CrossRef]

(c) (1)

(C) 2019 by the authors. Licensee MDPI, Basel, Switzerland. This article is an open access article distributed under the terms and conditions of the Creative Commons Attribution (CC BY) license (http://creativecommons.org/licenses/by/4.0/). 